\title{
Stop-mass prediction in naturalness scenarios within MSSM-25
}

\author{
Shehu S. AbdusSalam ${ }^{1(a),(b)}$ \\ (a) INFN, University of Rome "La Sapienza", Piazzale A. Moro 2, I-00185 Roma, Italy \\ (b) The Abdus Salam International Centre for Theoretical Physics, Strada Costiera 11, I-34151 \\ Trieste, Italy
}

\begin{abstract}
The "top-down" approach to minimal supersymmetric standard model (MSSM) phenomenology provides model-dependent indications for a natural stop mass. The approach is based on specific assumptions about the supersymmetry-breaking energy scale and parameters degeneracies. In order to determine robust predictions we update the stop-mass prediction within the MSSM with 25 parameters (MSSM-25) by including electroweak fine-tuning as "naturalness data" during the Bayesian fits of the parameters to experimental data. The approximately prior-independent results show that imposing naturalness, taken here to mean a $25 \%$ to $100 \%$ fine-tuning, predicts a $1-2 \mathrm{TeV}$ stop mass. The posterior distributions for the neutralinoproton cross sections indicate better prospects for probing the associated neutralino cold dark matter (CDM) with future upgrades of the detection facilities.
\end{abstract}

Introduction: Fitting the R-parity conserving MSSM-25 to pre-LHC experimental data predicts a now discovered [1, 2], Higgs boson mass and the, so far undiscovered, stop mass to be respectively $117-129 \mathrm{GeV}$ and $2-3 \mathrm{TeV}$ at $95 \%$ Bayesian credibility region [3, 4]. The SUSY-breaking parameters were simultaneously varied at the $\mathrm{TeV}$-scale, which the LHC is meant to probe, independent of hidden-sector physics, mediation mechanisms and with minimal renormalisation group running restrictions. In this article, we update the analysis in Refs. [3, 4], within our MSSM-25 programme [3, 4, 5, 6, 7, 8, 9], for addressing the implications of naturalness requirement on the stop mass prediction.

The studies on MSSM naturalness and its implication on the stop mass can be classified into two main groups. On the one hand, is the "top-down" approach which depends on various well-motivated but ad-hoc simplifications of the MSSM parameters (such as CMSSM, the constrained MSSM) or ad-hoc simplifications of the MSSM sparticle spectrum (the so-called simplified models) as, for instance, in Refs. [10, 11, 12, 13, 14, 15, 16, 17, $18,19]$. For this group, naturalness predicts that the lighter stop mass $m_{\tilde{t}_{1}}$ can be $m_{\tilde{t}_{1}} \lesssim$ $173 \mathrm{GeV}$ or $700 \mathrm{GeV} \lesssim m_{\tilde{t}_{1}}$ depending on whether the unification scale, $\Lambda_{G U T}$, is $\Lambda_{G U T} \sim$ $10^{16} \mathrm{GeV}$ or $\Lambda_{G U T} \sim 10 \mathrm{TeV}$ respectively. The "bottom-up" approach, on the other hand, depends solely on the full MSSM sparticle spectrum and couplings specified at

\footnotetext{
${ }^{1}$ Email: Shehu.AbdusSalam@roma1.infn.it
} 
the electroweak scale [20, 21, 22]. Both approaches involve either randomly generated "predictions" or ad-hoc parameters/spectrum simplifications for the effect of naturalness requirement on the stop mass.

In this article, we require that naturalness prediction for the stop mass should be assessed via unambiguous phenomenological frame and assumptions. For this purpose, so far, we find the Bayesian approach such as done in Refs. [3, 4] to be the most appropriate tool since it has a systematic procedure for checking the stability of conclusions with respect to the strength of data and assumptions. The electroweak fine-tuning (EWFT) measure, $\Delta_{E W}$, defined in Ref. [20], which provides a measure of fine-tuning given a full MSSM spectrum, and couplings at the electroweak scale and Bayesian techniques will be used for finding robust indication for natural $m_{\tilde{t}_{1}}$ within MSSM-25. We consider $\Delta_{E W}$ as the most appropriate for our analysis given the match between its construction and the MSSM-25 parameterisation procedures. Both were developed as purely electroweak scalebased phenomena independent of SUSY-breaking physics and parameters renormalisation group running. The measures defined in Refs. [23, 24] depend on the parameters and the restrictions with which they usually come. The $\Delta_{E W}$ naturalness requirement cuts will be imposed as a constraint while exploring the MSSM-25 parameter space unlike via the marginalisation procedure as in Refs. [23, 24, 25, 26].

The electroweak fine-tuning measure: The fine-tuning measure is based on the minimisation of the 1-loop corrected potential energy, $V+\Delta V$, of the Higgs boson fields which leads to

$$
\frac{m_{Z}^{2}}{2}=\frac{m_{H_{d}}^{2}+\Sigma_{d}^{d}-\left(m_{H_{u}}^{2}+\Sigma_{u}^{u}\right) \tan ^{2} \beta}{\tan ^{2} \beta-1}-\mu^{2}
$$

where $\Sigma_{u}^{u}$ and $\Sigma_{d}^{d}$ are radiative corrections that arise from the derivatives of $\Delta V$ evaluated at the minimum $[20,21] \cdot m_{H_{u}}$ and $m_{H_{d}}$ are respectively the up-type and down-type Higgs doublet mass parameters, $\tan \beta=\left\langle H_{d}\right\rangle /\left\langle H_{u}\right\rangle$ is the ratio of their vacuum expectation values. $\mu$ represents the Higgs doublets mixing parameter. Naturalness requires each term in the right hand side of Eq. 1 to be comparable to $m_{Z}^{2} / 2$. The definition

$$
\Delta_{E W} \equiv \max _{i}\left(C_{i}\right) /\left(m_{Z}^{2} / 2\right)
$$

accommodates the fact that for obtaining a natural value of $m_{Z}$ then the terms $C_{i}$, with $i=$ $H_{d}, H_{u}, \mu, \Sigma_{u}^{u}(k), \Sigma_{d}^{d}(k)$, where $k$ denotes the various particles and sparticles contributions, must have an order $m_{Z}^{2} / 2$ absolute values. We use only the contributions (the case $i=$ $\left.\tilde{t}_{1,2}, \tilde{b}_{1,2}\right)$ from terms that couple the most to the Higgs sector:

$$
\begin{aligned}
C_{\mu} & =\left|-\mu^{2}\right|, \\
C_{H_{u}} & =\left|-m_{H_{u}}^{2} \tan ^{2} \beta /\left(\tan ^{2} \beta-1\right)\right|, \\
C_{H_{d}} & =\left|m_{H_{d}}^{2} /\left(\tan ^{2} \beta-1\right)\right|, \\
C_{\Sigma_{d}^{d}} & =\left|\Sigma_{d}^{d} /\left(\tan ^{2} \beta-1\right)\right|, \\
C_{\Sigma_{u}^{u}} & =\left|-\Sigma_{u}^{u} \tan ^{2} \beta /\left(\tan ^{2} \beta-1\right)\right|, \\
\Sigma_{d, u}^{d, u} & =\Sigma_{i}\left|\Sigma_{d, u}^{d, u}(i)\right| .
\end{aligned}
$$

The expressions for $\Sigma_{d, u}^{d, u}(i)$ are given in the Appendix. 
Bayesian global fit method and naturalness: The cut applied on the EWFT measure Eq. 2 to restrict its values to acceptable level were considered as "naturalness data" for the Bayesian fits (with linear and logarithmic priors on the parameters) of the MSSM-25 to data (listed in Eq. 7). Any prior-independent result ${ }^{1}$ or posterior distribution concerning the stop mass obtained from the Bayesian fits will constitute a robust "naturalness prediction" within the MSSM-25. For completeness, we shall introduce Bayes' theorem first before describing the procedure for the fits. Bayes' theorem states that given a model hypothesis, $\mathcal{H}$, with parameters, $\underline{\theta}$, and a set of data, $\underline{d}$, for constraining the model then

$$
p(\underline{\theta} \mid \underline{d}, \mathcal{H})=\frac{p(\underline{d} \mid \underline{\theta}, \mathcal{H}) p(\underline{\theta} \mid \mathcal{H})}{p(\underline{d} \mid \mathcal{H})}
$$

where $p(\underline{\theta} \mid \mathcal{H})$ is the prior probability distribution which provides information about the model parameters before the data $\underline{d}$ is taken into consideration. The information about the model in light of the data is represented by the probability distribution $p(\underline{d} \mid \underline{\theta}, \mathcal{H})$ or the likelihood of the model given the data. $p(\underline{\theta} \mid \underline{d}, \mathcal{H})$ is the probability distribution of the model parameters given the data.

The Bayesian fit procedure: The fits were done within the context, $\mathcal{H}$, that Nature is supersymmetric as captured by MSSM-25 and that the neutralino lightest supersymmetric particle (LSP) explains at least partially the observed cold dark matter (CDM) relic density [5]. The MSSM-25 parameters are

$\underline{\theta}=\left\{M_{1,2,3} ; \quad m_{\tilde{f}_{Q, U, D, L, E}}^{3 r d g e n}, m_{\tilde{f}_{Q, U, D, L, E}}^{1 s t / 2 n d g e n} ; A_{t, b, \tau, \mu=e}, m_{H_{u, d}}^{2}, \tan \beta ; m_{Z}, m_{t}, m_{b}, \alpha_{e m}^{-1}, \alpha_{s}\right\}$

where $M_{1}, M_{2}$ and $M_{3}$ are the gaugino mass parameters (allowed in the range -4 to 4 $\mathrm{TeV}$ ) and $m_{\tilde{f}}$ are the sfermion mass parameters (allowed in the range $100 \mathrm{GeV}$ to 4 $\mathrm{TeV}) . A_{t, b, \tau, \mu=e} \in[-8,8] \mathrm{TeV}$ represents the trilinear scalar couplings, while the Higgssector parameters are specified by the two Higgs doublet masses $m_{H_{1}}^{2}, m_{H_{2}}^{2}$ (with $m^{2} \in$ $\left.\operatorname{sign}(m)[-4,4]^{2} \mathrm{TeV}^{2}\right)$, the ratio of the vacuum expectation values $\tan \beta=\left\langle H_{2}\right\rangle /\left\langle H_{1}\right\rangle$ (allowed between 2 and 60) and $\operatorname{sign}(\mu)$ is the sign of the Higgs doublets mixing parameter (allowed to be randomly \pm 1 .) The mass of the Z-boson, $m_{Z}=91.1876 \pm 0.0021$; the top quark mass, $m_{t}=172.6 \pm 1.4 \mathrm{GeV}$, the bottom quark mass, $m_{b}=4.2 \pm 0.07 \mathrm{GeV}$, the electromagnetic coupling, $\alpha_{e m}^{-1}=127.918 \pm 0.018$, and the strong interaction coupling, $\alpha_{s}=0.1172 \pm 0.002$, were all set to vary in a Gaussian manner with central values and deviations according to the experimental results. The two Bayesian global fits were done with linear and logarithmic prior assumptions for the SUSY-breaking parameters.

Adding the EWFT measure $\Delta_{E W}$ to the list of data makes

$$
\underline{d}=d_{\text {pre-LHC }} \oplus d_{\Delta_{E W}}=\left\{\mu_{i}, \sigma_{i}\right\} \oplus\left\{\Delta_{E W}^{-1} \geq 5 \%\right\} .
$$

Here $d_{\text {pre-LHC }}{ }^{2}$ represents the Higgs boson mass, the electroweak physics, B-physics and the cold dark matter relic density observables, represented by their corresponding central

\footnotetext{
${ }^{1}$ If the posterior distribution of a quantity (for example the stop mass) coming from a fit with a flat prior on the parameters is widely different from that coming from a fit with a logarithmic prior then the result (the stop mass distribution) is said to be prior-dependent. Otherwise the result may be considered to be approximately or fully prior-independent.

${ }^{2}$ We are going to maintain this notation despite the fact that the Higgs boson mass is no longer a "pre-LHC" observable.
} 


\begin{tabular}{|cl||cl|}
\hline Observable & Constraint & Observable & Constraint \\
\hline$m_{W}[\mathrm{GeV}]$ & $80.399 \pm 0.027[32]$ & $A^{l}=A^{e}$ & $0.1513 \pm 0.0021[33]$ \\
$\Gamma_{Z}[\mathrm{GeV}]$ & $2.4952 \pm 0.0025[33]$ & $A^{b}$ & $0.923 \pm 0.020[33]$ \\
$\sin ^{2} \theta_{\text {eff }}^{l e p}$ & $0.2324 \pm 0.0012[33]$ & $A^{c}$ & $0.670 \pm 0.027[33]$ \\
$\delta a_{\mu}$ & $(30.2 \pm 9.0) \times 10^{10}[34,35]$ & $B r\left(B \rightarrow X_{s} \gamma\right)$ & $(3.55 \pm 0.42) \times 10^{4}[36]$ \\
$R_{l}^{0}$ & $20.767 \pm 0.025[33]$ & $B r\left(B_{s} \rightarrow \mu^{+} \mu^{-}\right)$ & $3.2_{-1.2}^{+1.5} \times 10^{-9}[37]$ \\
$R_{b}^{0}$ & $0.21629 \pm 0.00066[33]$ & $R_{\Delta M_{B_{s}}}$ & $0.85 \pm 0.11[38]$ \\
$R_{c}^{0}$ & $0.1721 \pm 0.0030[33]$ & $R_{B r\left(B_{u} \rightarrow \tau \nu\right)}$ & $1.26 \pm 0.41[39,40,41]$ \\
$A_{\mathrm{FB}}^{b}$ & $0.0992 \pm 0.0016[33]$ & $\Delta_{0-}$ & $0.0375 \pm 0.0289[42]$ \\
$A_{\mathrm{FB}}^{c}$ & $0.0707 \pm 0.035[33]$ & $\Omega_{C D M} h^{2}$ & $0.11 \pm 0.02[43]$ \\
& & $m_{h}$ & $125.6 \pm 3.0[\mathrm{GeV}][44,45]$ \\
\hline
\end{tabular}

Table 1: Summary for the central values and errors for the Higgs boson mass, the electroweak physics observables, B-physics observables and cold dark matter relic density constraints.

values $\left(\mu_{i}\right)$ and errors $\left(\sigma_{i}\right)$ where $i=1,2, \ldots$, enumerates the observables as summarised in Table 1 and the list:

$$
\begin{aligned}
\underline{O}= & \left\{m_{W}, \sin ^{2} \theta_{e f f}^{l e p}, \Gamma_{Z}, \delta a_{\mu}, R_{l}^{0}, A_{f b}^{0, l}, A^{l}=A^{e}, R_{b, c}^{0}, A_{f b}^{b, c}, A^{b, c}, B R\left(B \rightarrow X_{s} \gamma\right),\right. \\
& \left.B R\left(B_{s} \rightarrow \mu^{+} \mu^{-}\right), \Delta_{0-}, R_{B R\left(B_{u} \rightarrow \tau \nu\right)}, R_{\Delta M_{B_{s}}}, \Omega_{C D M} h^{2}, m_{h}, \Delta_{E W}^{-1} \geq 5 \%\right\} .
\end{aligned}
$$

The SUSY spectrum calculator SOFTSUSY [27] was used for computing the spectra, mixing angles and couplings from the input soft SUSY-breaking parameters, MICROMEGAs [28] for computing the neutralino CDM relic density and the anomalous magnetic moment of the muon $\delta a_{\mu}$, SuPERIso [29] for predicting the branching ratios $B R\left(B_{s} \rightarrow \mu^{+} \mu^{-}\right), B R(B \rightarrow s \gamma)$ and the isospin asymmetry, $\Delta_{0-}$, in the decays $B \rightarrow K^{*} \gamma$, and SUSYPOPE $[30,31]$ for computing precision observables that include the $W$-boson mass $m_{W}$, the effective leptonic mixing angle variable $\sin ^{2} \theta_{\text {eff }}^{\text {lep }}$, the total $Z$-boson decay width, $\Gamma_{Z}$, and the other electroweak observables whose experimentally determined central values and associated errors are summarised in Table 1. The posterior probability is thus given by Bayes' theorem, Eq. 4, as

$$
\begin{gathered}
p(\underline{\theta} \mid \underline{d}, \mathcal{H})=L_{\Delta_{E W}} L_{C D M}(x) \prod_{i} \frac{e^{\left[-\left(O_{i}-\mu_{i}\right)^{2} / 2 \sigma_{i}^{2}\right]}}{\sqrt{2 \pi \sigma_{i}^{2}}} \frac{p(\underline{\theta} \mid \mathcal{H})}{p(\underline{d} \mid \mathcal{H})} ; \\
L_{\Delta_{E W}}=\left\{\begin{array}{ll}
1, & \text { if } \Delta_{E W}^{-1} \geq 5 \% \\
0, & \text { if } \Delta_{E W}^{-1}<5 \%
\end{array}, \quad L_{C D M}(x)= \begin{cases}1 /\left(y+\sqrt{\pi s^{2} / 2}\right), & \text { if } x<y \\
e^{\left[-(x-y)^{2} / 2 s^{2}\right] /\left(y+\sqrt{\pi s^{2} / 2}\right),} & \text { if } x \geq y\end{cases} \right.
\end{gathered}
$$

where the index $i$ run over the different experimental observables (data) other than the CDM relic density, $x$ represents the predicted value of the neutralino CDM relic density, $y=0.11$ is the WMAP central value quoted in Table 1 and $s=0.02$ the inflated error. The likelihood contribution coming from the CDM relic density is given by $L_{C D M}(x)$ which is purely Gaussian when the predicted relic density $x$ is greater than the experimental central 
value $y=0.11$ thus imposing penalisation for CDM over-production. No penalisation is imposed when $x<y$. This way the fits allow for the possibility for multicomponent CDM such that the non-neutralino LSP component(s) will account for the relic density deficit. We used the MultiNest $[46,47]$ package that implements nested sampling algorithm [48] for exploring the MSSM-25 parameters by including the requirement of a minimal finetuning, $\Delta_{E W} \leq 20$, in the likelihood function.

Another set of fits were done with $\Delta_{E W} \leq 4$, besides the $\Delta_{E W} \leq 20$ one, as the minimally possible fine-tuning in the sense of the upper bound of $\Delta_{E W}=2 \pm 2$. This is for the purpose of checking the stability of the stop mass posterior distribution with respect to tighter $\Delta_{E W}$ (less fine-tuning) cuts. The reason for considering $\Delta_{E W}=2 \pm 2$ is as follows. In principle, the model point with no fine-tuning at all, according to the chosen measure, will have $\Delta_{E W} \rightarrow 0$. But such a model point with infinitesimally small finetuning is difficult to obtain in practice. Therefore we suppose or define $\Delta_{E W}=2$ which corresponds to a fine-tuning not worse than $50 \%$ to be a reasonable minimum within a $100 \%$ (of $\Delta_{E W}=2$ ) theoretical error allowance i.e. $\delta \Delta_{E W}= \pm 2$. With these assumptions, then $\Delta_{E W} \leq 4$ is practically the most strong naturalness cut we could impose for the fits.

The $\Delta_{E W} \leq 4$ fits were done with a relaxed Higgs boson mass constraints by allowing $m_{h}<122 \mathrm{GeV}$ but with Gaussian suppressed probabilities around $m_{h}=125.6 \pm 3.0 \mathrm{GeV}$. For the first set of fits with $\Delta_{E W} \leq 20$ all MSSM-25 points with $m_{h}<122 \mathrm{GeV}$ were discarded. This little but significant change on the Higgs boson mass constraint is used explicitly to point to the effects of the fine-tuning versus $m_{h}$ cuts on the stop mass (see the tails of the $m_{h}$ distributions in Fig. 2 and the stop mass distributions in Fig. 1.)

Sparticle mass, $\tilde{\chi}_{1}^{0}$ relic density and $B R\left(B_{s} \rightarrow \mu^{+} \mu^{-}\right)$predictions: Most of the posterior distributions from both fits with $\Delta_{E W} \leq 20$ and $\Delta_{E W} \leq 4$ have a prior-dependent feature except, as is expected from Eq. 1, for the neutralino, chargino and the lighter stops masses. The approximately prior-independent tendency for $m_{\tilde{t}_{1}}, m_{\tilde{\chi}_{1,2}^{0}}$ and $m_{\tilde{\chi}_{1}^{ \pm}}$ distributions can be seen in Fig. 1. It can be deduced from the results that naturalness imposes upper bounds on $m_{\tilde{\chi}_{1,2}^{0}}$ and $m_{\tilde{\chi}_{1}^{ \pm}}$. This is because of the tree-level fine-tuning restriction on the $\mu$ parameter to be near the Z-boson mass, $m_{Z}$. The effect on $m_{\tilde{t}_{1}}$ and $m_{\tilde{g}}$ are at the 1-loop and 2-loop levels respectively. It is the Higgs boson mass constraint $m_{h} \sim$ $125 \mathrm{GeV}$ that pulls the fits in fixing $m_{\tilde{t}_{1}} \sim 1-2 \mathrm{TeV}$ to be approximately prior independent as shown on the first row of Fig. 1. Relaxing this constraint has the effect of lowering the stop mass magnitudes as shown in the second row of Fig. 1. Thus the $m_{h} \sim 125 \mathrm{GeV}$ and the EWFT constraint corner the stops to $m_{\tilde{t}_{1}} \sim 1-2 \mathrm{TeV}$. This region is well above the ATLAS and CMS bounds in Refs. [49, 50, 51, 52, 53, 54, 55, 56, 57, 58, 59, 60, 61, 62]. The bits of parameter space with very light $\left(\Delta m \lesssim m_{\pi^{ \pm}}\right)$to intermediate $\left(m_{\pi^{ \pm}} \lesssim \Delta m \lesssim 300\right.$ $\mathrm{MeV}$ ) chargino-neutralino mass difference $\Delta m=m_{\chi_{1}^{ \pm}}-m_{\chi_{1}^{0}}$ up to around $5 \mathrm{GeV}$ (see Fig. 2 for the distributions of $\Delta m$ ) are constrained by LEP and LHC results such as in Refs. [63, 64, 65, 66]. However, the effect of the cuts on the posterior distribution is insignificant.

The neutralino mass-eigenstate is made of bino $\tilde{b}$, wino $\tilde{w}^{3}$ and Higgsinos $\tilde{H}_{1,2}$ combination:

$$
\tilde{\chi}_{1}^{0}=N_{11} \tilde{b}+N_{12} \tilde{w}^{3}+N_{13} \tilde{H}_{1}^{0}+N_{14} \tilde{H}_{2}^{0}, \quad \sum_{i=1,2,3,4}\left(N_{1 i}\right)^{2}=1
$$



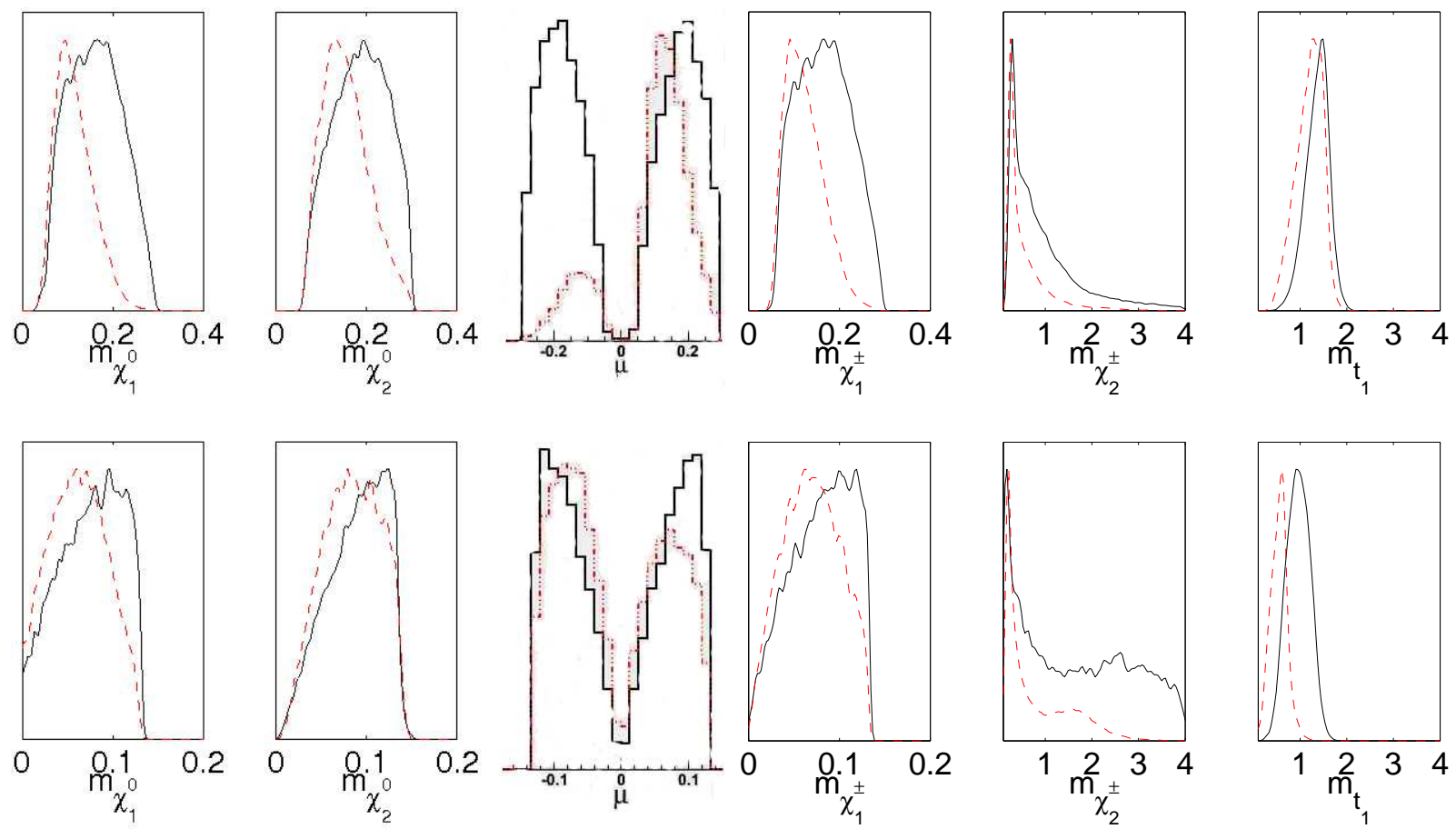

Figure 1: (First row:) The plots show the posterior distributions of sparticles with an approximately prior-independent feature over limited regions from the $\Delta_{E W} \leq 20$ fit. Dotted(solid) curves are for the $\log$ (flat) prior fits of the MSSM-25. All the masses are in TeV units. (Second row:) The plots are the same as in the first row but from the $\Delta_{E W} \leq 4 \mathrm{fit}$. Note that the $m_{\tilde{t}_{1}}$ distribution is now prior-dependent due to the relaxation of the Higgs-boson mass constraint. The $m_{\tilde{\chi}_{1,2}^{0}}$ and $m_{\tilde{\chi}_{1}^{ \pm}}$are now preferred to be lighter relative to the $\Delta_{E W} \leq 20$ results because of the tighter constraint on the Higgs bosons mixing parameter $\mu$ by imposing $\Delta_{E W} \leq 4$.

where $N_{1 i}$ with $i=1,2,3,4$ are coefficient depending on soft-SUSY breaking terms [67]. In Fig. 2, $\left(1-Z_{g}\right)$ where $Z_{g}=\left|N_{11}\right|^{2}+\left|N_{22}\right|^{2}$ quantifies the nature of the neutralino to be dominantly Higgsino- or gaugino-like for $Z_{g}$ approximately equal to unity or zero respectively. The neutralino-chargino mass quasi-degeneracy (see the $\Delta m$ plots in Fig. 2) together with the Higgsino-nature of the neutralino enhances the primordial co-annihilations of the neutralino CDM to the effect that the relic density is much lower than the observed value around 0.1. The remaining observed relic density has to be accounted for by a non-neutralino CDM component(s). The posterior distribution for the branching ratio of the decay $B_{s} \rightarrow \mu^{+} \mu^{-}$is also approximately prior-independent near the standard model value ${ }^{3}$.

Prospects for direct detection of the $\tilde{\chi}_{1}^{0}$ CDM: Here we address the prospects of direct detection of the neutralino part of CDM. The spin-independent neutralino-proton cross section, $\sigma_{\chi_{1}^{0} p}^{S I}$, distributions against the neutralino mass are shown in Fig. 3. In order to account for the fact that the neutralino makes up only part of the dark matter relics, the cross sections are rescaled by a factor $\xi=\Omega_{\chi_{1}^{0}} h^{2} / 0.11$ as suggested in Ref. [68]. It can be seen from Fig. 3 that the past/current dark matter direct detection results such as

\footnotetext{
${ }^{3}$ We shall analyse this decay in a separate report.
} 

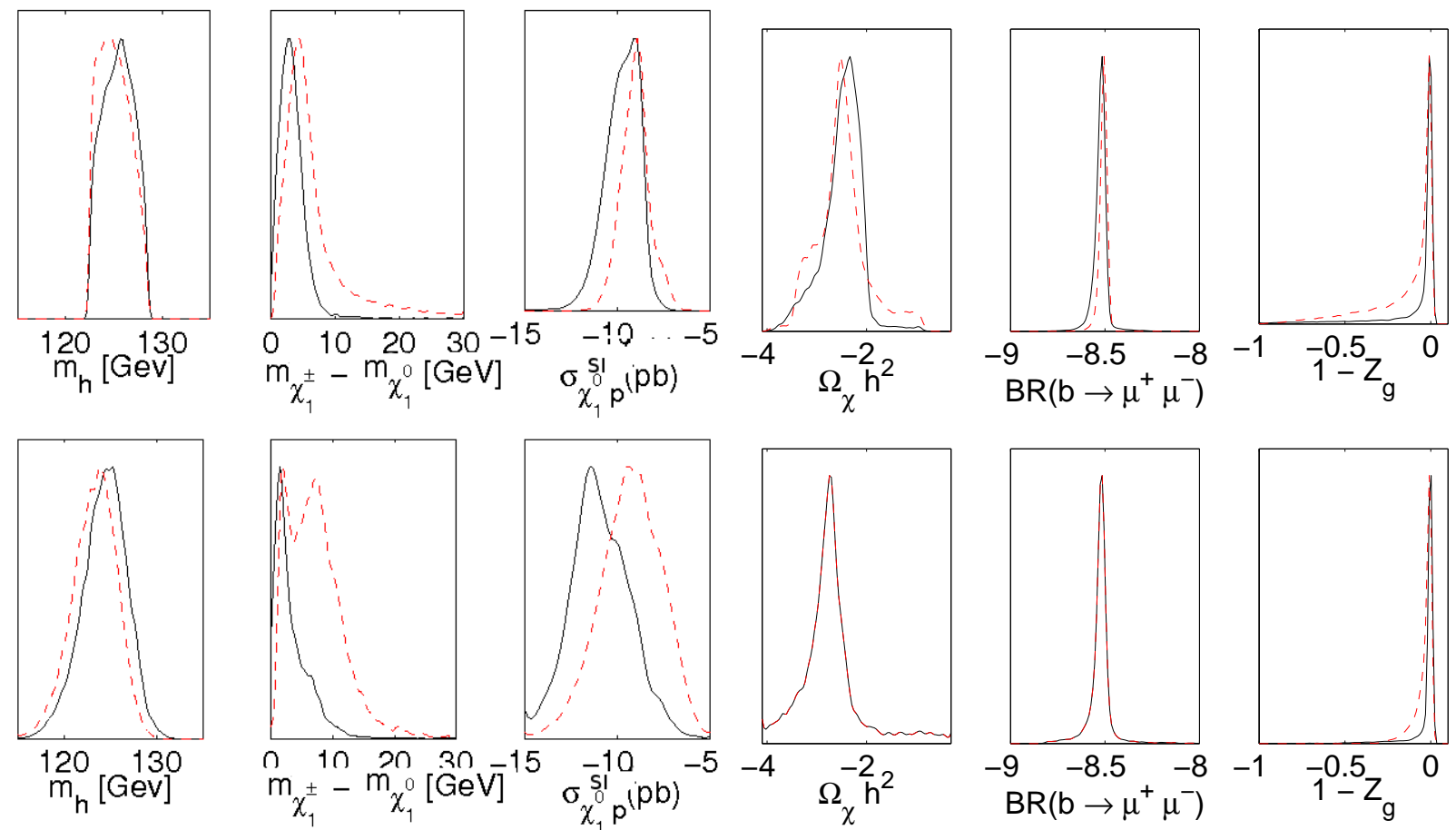

Figure 2: (First row:) The plots show the $\Delta_{E W} \leq 20$ posterior distributions for the Higgs boson mass $m_{h}$, the mass difference $\Delta m=m_{\chi_{1}^{ \pm}}-m_{\chi_{1}^{0}}$, the spin-independent neutralino-proton cross section, $\sigma_{\chi_{1}^{0} p}^{S I}$, the neutralino relic density $\Omega_{\chi_{1}^{0}} h^{2}$, the branching ratio of the decay $B_{s} \rightarrow \mu^{+} \mu^{-}$, and the measure for quantifying the nature of the neutralino components $1-Z_{g}$ described in the text. All the x-axes other than the masses are in the log-10 bases. (Second row:) The plots are the same as in the first row but from the $\Delta_{E W} \leq 4$ fits.

from CDMS [69] and XENON [70] collaborations will hardly probe the interesting regions within the MSSM-25. The result from the LUX experimental collaboration [71] is a bit different given that the $90 \%$ confidence limit on the cross section crosses over a small but relatively more significant probability mass of the favoured region compared to the earlier bounds. The prospect for detection is much enhanced for future upgrades of the detectors such as the ton-scale liquid Xenon detector [72].

Conclusions: We have addressed the question of SUSY naturalness within R-parity conserving MSSM-25. We use naturalness requirements as a "fine-tuning data" on the same footing as, for example, the CDM relic density data in a Bayesian statistical method for fitting the MSSM-25 parameters. Doing this allows for a robust assessment of naturalness criterion strength in constraining the SUSY parameters. Two separate fittings were performed with naturalness data $\Delta_{E W} \leq 20$ and $\Delta_{E W} \leq 4$. The results show that the combination of Higgs-boson mass constraint and naturalness requirements corners the stop mass to $1-2 \mathrm{TeV}$. This reduction relative to the pre-LHC prediction of $2-3 \mathrm{TeV}$ [4] is due to the naturalness pull. The stop prediction is associated with an upper bounds on $m_{\tilde{\chi}_{1,2}^{0}}$ and $m_{\tilde{\chi}_{1}^{ \pm}}$to be sub-half TeV. The LUX-2013 experiment is beginning to probe slightly the favoured region of parameter space. The prospects for $\tilde{\chi}_{1}^{0} \mathrm{CDM}$ discovery look better with future upgrades of the direct detection facilities. 

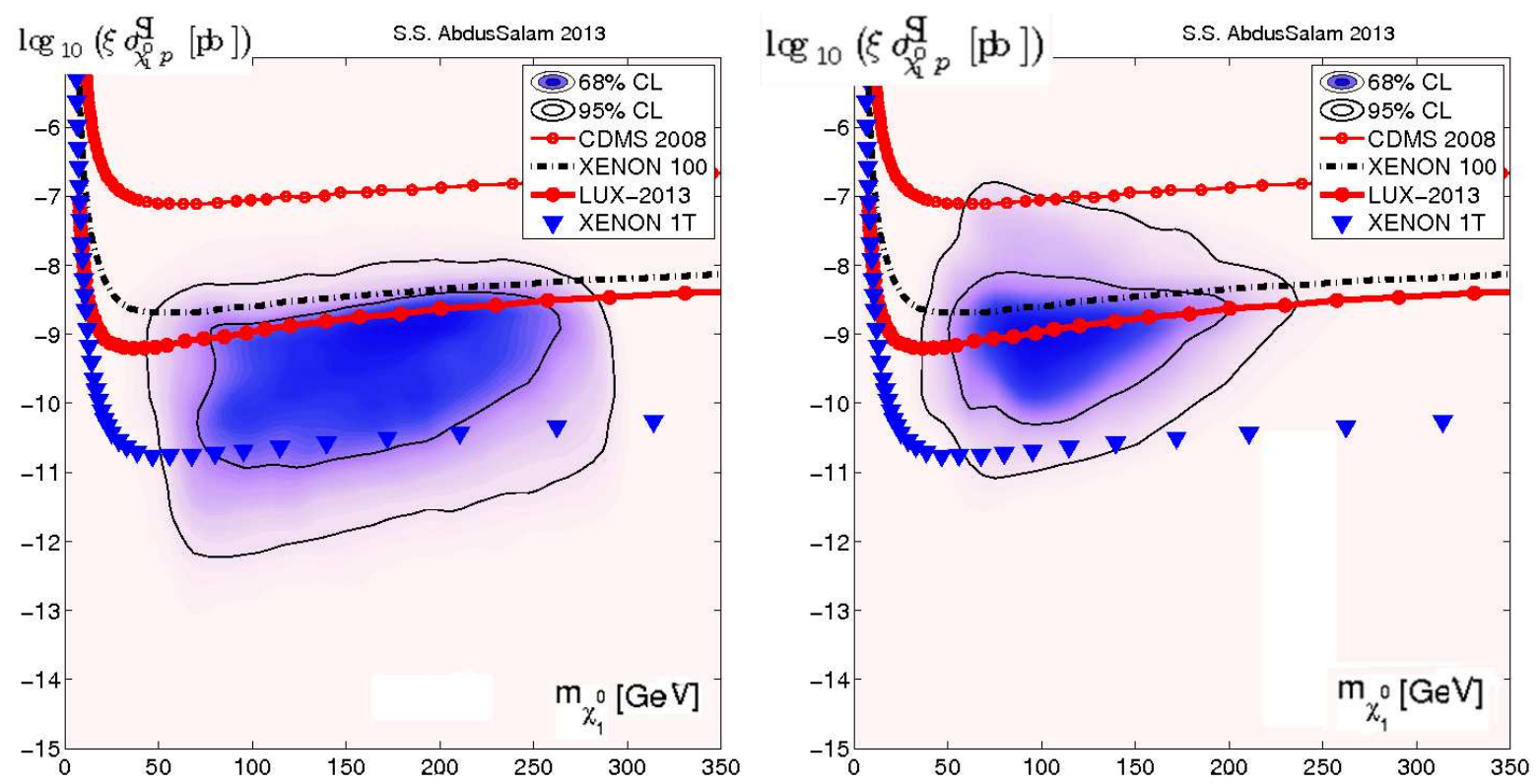

Figure 3: The plots show the posterior distributions of the neutralino-proton spin-independent scattering cross section $\sigma_{\chi_{1}^{0} p}^{S I}$ (reduced by the factor $\xi=\Omega_{C D M} h^{2} / 0.11$ described in the text) for the MSSM-25 with linear (left) and log (right) prior measures. The CDMS-2008 90\% confidence level [69], XENON-100 [70], LUX-2013 [71] and the future-projected XENON-1TN [72] upper bound constraints are shown as summarised by the legend of the plots. The solid contour lines show the $68 \%$ and $95 \%$ Bayesian credibility regions.

Acknowledgement: The author acknowledges useful discussions with H. Baer, F. Quevedo, G. Villadoro and L. Velasco-Sevilla. This work was performed using the Darwin Supercomputer of the University of Cambridge High Performance Computing Service (http://www.hpc.cam.ac.uk/ provided by Dell Inc. using Strategic Research Infrastructure Funding from the Higher Education Funding Council for England and funding from the Science and Technology Facilities Council.

Appendix: The formulae for $\Sigma_{u, d}^{u, d}(i), i=\tilde{t}_{1,2}, \tilde{b}_{1,2}$ At one-loop the $\Sigma_{u, d}^{u, d}$ get both particle and sparticle contributions [73, 74] but here we considered only the $\Sigma_{u}^{u}\left(\tilde{t}_{1,2}, \tilde{b}_{1,2}\right)$ ones. For the stops,

$$
\begin{gathered}
\Sigma_{u}^{u}\left(\tilde{t}_{1,2}\right)=\frac{3}{16 \pi^{2}} F\left(m_{\tilde{t}_{1,2}}^{2}\right) \times\left[f_{t}^{2}-g_{Z}^{2} \mp \frac{f_{t}^{2} A_{t}^{2}-8 g_{Z}^{2}\left(\frac{1}{4}-\frac{2}{3} x_{W}\right) \Delta_{t}}{m_{\tilde{t}_{2}}^{2}-m_{\tilde{t}_{1}}^{2}}\right] \text { and } \\
\Sigma_{d}^{d}\left(\tilde{t}_{1,2}\right)=\frac{3}{16 \pi^{2}} F\left(m_{\tilde{t}_{1,2}}^{2}\right)\left[g_{Z}^{2} \mp \frac{f_{t}^{2} \mu^{2}+8 g_{Z}^{2}\left(\frac{1}{4}-\frac{2}{3} x_{W}\right) \Delta_{t}}{m_{\tilde{t}_{2}}^{2}-m_{\tilde{t}_{1}}^{2}}\right]
\end{gathered}
$$

where $\Delta_{t}=\left(m_{\tilde{t}_{L}}^{2}-m_{\tilde{t}_{R}}^{2}\right) / 2+M_{Z}^{2} \cos 2 \beta\left(\frac{1}{4}-\frac{2}{3} x_{W}\right), g_{Z}^{2}=\left(g^{2}+g^{\prime 2}\right) / 8, x_{W} \equiv \sin ^{2} \theta_{W}$ and $F\left(m^{2}\right)=m^{2}\left(\log \left(m^{2} / Q^{2}\right)-1\right)$, with $Q^{2}=m_{\tilde{t}_{1}} m_{\tilde{t}_{2}} \cdot m_{\tilde{t}_{1,2}}$ are computed at tree-level. For 
the bottom-squarks,

$$
\begin{aligned}
& \Sigma_{u}^{u}\left(\tilde{b}_{1,2}\right)=\frac{3}{16 \pi^{2}} F\left(m_{\tilde{b}_{1,2}}^{2}\right)\left[g_{Z}^{2} \mp \frac{f_{b}^{2} \mu^{2}-8 g_{Z}^{2}\left(\frac{1}{4}-\frac{1}{3} x_{W}\right) \Delta_{b}}{m_{\tilde{b}_{2}}^{2}-m_{\tilde{b}_{1}}^{2}}\right] \text { and } \\
& \Sigma_{d}^{d}\left(\tilde{b}_{1,2}\right)=\frac{3}{16 \pi^{2}} F\left(m_{\tilde{b}_{1,2}}^{2}\right)\left[f_{b}^{2}-g_{Z}^{2} \mp \frac{f_{b}^{2} A_{b}^{2}-8 g_{Z}^{2}\left(\frac{1}{4}-\frac{1}{3} x_{W}\right) \Delta_{b}}{m_{\tilde{b}_{2}}^{2}-m_{\tilde{b}_{1}}^{2}}\right]
\end{aligned}
$$

where $\Delta_{b}=\left(m_{\tilde{b}_{L}}^{2}-m_{\tilde{b}_{R}}^{2}\right) / 2+M_{Z}^{2} \cos 2 \beta\left(\frac{1}{4}-\frac{1}{3} x_{W}\right) \cdot m_{\tilde{b}_{1,2}}$ are computed at tree-level.

\section{References}

[1] ATLAS Collaboration, G. Aad et al., "Observation of a new particle in the search for the Standard Model Higgs boson with the ATLAS detector at the LHC," Phys.Lett. B716 (2012) 1-29, arXiv:1207.7214 [hep-ex].

[2] CMS Collaboration, S. Chatrchyan et al., "Observation of a new boson at a mass of $125 \mathrm{GeV}$ with the CMS experiment at the LHC," Phys.Lett. B716 (2012) 30-61, arXiv:1207.7235 [hep-ex].

[3] S. S. AbdusSalam, "The Full 24-Parameter MSSM Exploration," AIP Conf.Proc. 1078 (2009) 297-299, arXiv:0809.0284 [hep-ph].

[4] S. S. AbdusSalam, B. C. Allanach, F. Quevedo, F. Feroz, and M. Hobson, "Fitting the Phenomenological MSSM," Phys.Rev. D81 (2010) 095012, arXiv:0904.2548 [hep-ph].

[5] S. AbdusSalam and F. Quevedo, "Cold Dark Matter Hypotheses in the MSSM," Phys.Lett. B700 (2011) 343-350, arXiv: 1009.4308 [hep-ph].

[6] S. AbdusSalam, "Can the LHC rule out the MSSM?," Phys.Lett. B705 (2011) 331-336, arXiv:1106.2317 [hep-ph].

[7] S. AbdusSalam, B. Allanach, H. Dreiner, J. Ellis, U. Ellwanger, et al., "Benchmark Models, Planes, Lines and Points for Future SUSY Searches at the LHC," Eur.Phys.J. C71 (2011) 1835, arXiv:1109.3859 [hep-ph].

[8] S. S. AbdusSalam and D. Choudhury, "Higgs boson discovery versus sparticles prediction: Impact on the pMSSM's posterior samples from a Bayesian global fit," arXiv:1210.3331 [hep-ph].

[9] S. S. AbdusSalam, "LHC-7 supersymmetry search interpretation within the pMSSM," Phys.Rev. D87 (2013) 115012, arXiv: 1211.0999 [hep-ph].

[10] B. de Carlos and J. Casas, "One loop analysis of the electroweak breaking in supersymmetric models and the fine tuning problem," Phys.Lett. B309 (1993) 320-328, arXiv:hep-ph/9303291 [hep-ph] .

[11] K. L. Chan, U. Chattopadhyay, and P. Nath, "Naturalness, weak scale supersymmetry and the prospect for the observation of supersymmetry at the Tevatron and at the CERN LHC," Phys.Rev. D58 (1998) 096004, arXiv:hep-ph/9710473 [hep-ph] .

[12] S. Dimopoulos and G. Giudice, "Naturalness constraints in supersymmetric theories with nonuniversal soft terms," Phys.Lett. B357 (1995) 573-578, arXiv:hep-ph/9507282 [hep-ph].

[13] V. Berezinsky, A. Bottino, J. R. Ellis, N. Fornengo, G. Mignola, et al., "Neutralino dark matter in supersymmetric models with nonuniversal scalar mass terms," Astropart.Phys. 5 (1996) 1-26, arXiv:hep-ph/9508249 [hep-ph].

[14] S. Cassel, D. Ghilencea, S. Kraml, A. Lessa, and G. Ross, "Fine-tuning implications for complementary dark matter and LHC SUSY searches," JHEP 1105 (2011) 120, arXiv:1101.4664 [hep-ph].

[15] D. Ghilencea and G. Ross, "The fine-tuning cost of the likelihood in SUSY models," Nucl.Phys. B868 (2013) 65-74, arXiv: 1208.0837 [hep-ph].

[16] T. Kobayashi, H. Terao, and A. Tsuchiya, "Fine-tuning in gauge mediated supersymmetry breaking models and induced top Yukawa coupling," Phys.Rev. D74 (2006) 015002, arXiv:hep-ph/0604091 [hep-ph].

[17] A. Arvanitaki, N. Craig, S. Dimopoulos, and G. Villadoro, "Mini-Split," JHEP 1302 (2013) 126, arXiv:1210.0555 [hep-ph].

[18] N. Arkani-Hamed, A. Gupta, D. E. Kaplan, N. Weiner, and T. Zorawski, "Simply Unnatural Supersymmetry," arXiv:1212.6971 [hep-ph].

[19] J. L. Feng, "Naturalness and the Status of Supersymmetry," Ann.Rev.Nucl.Part.Sci. 63 (2013) 351-382, arXiv:1302.6587 [hep-ph].

[20] H. Baer, V. Barger, P. Huang, A. Mustafayev, and X. Tata, "Radiative natural SUSY with a 125 GeV Higgs boson," Phys.Rev.Lett. 109 (2012) 161802, arXiv:1207.3343 [hep-ph].

[21] H. Baer, V. Barger, P. Huang, D. Mickelson, A. Mustafayev, et al., "Radiative natural supersymmetry: Reconciling electroweak fine-tuning and the Higgs boson mass," Phys.Rev. D87 (2013) 115028, arXiv:1212.2655 [hep-ph].

[22] H. Baer, V. Barger, P. Huang, D. Mickelson, A. Mustafayev, et al., "Post-LHC7 fine-tuning in the mSUGRA/CMSSM model with a 125 GeV Higgs boson," Phys.Rev. D87 no. 3, (2013) 035017, arXiv:1210.3019 [hep-ph].

[23] R. Barbieri and G. Giudice, "Upper Bounds on Supersymmetric Particle Masses," Nucl.Phys. B306 (1988) 63. 
[24] J. R. Ellis, K. Enqvist, D. V. Nanopoulos, and F. Zwirner, "Observables in Low-Energy Superstring Models," Mod. Phys. Lett. A1 (1986) 57.

[25] M. Cabrera, J. Casas, and R. Ruiz de Austri, "Bayesian approach and Naturalness in MSSM analyses for the LHC," JHEP 0903 (2009) 075, arXiv:0812.0536 [hep-ph].

[26] D. Ghilencea, "SUSY naturalness without prejudice," arXiv:1311.6144 [hep-ph].

[27] B. Allanach, "SOFTSUSY: a program for calculating supersymmetric spectra," Comput.Phys.Commun. 143 (2002) 305-331, arXiv:hep-ph/0104145 [hep-ph].

[28] G. Belanger, F. Boudjema, A. Pukhov, and A. Semenov, "Dark matter direct detection rate in a generic model with micrOMEGAs 2.2," Comput.Phys.Commun. 180 (2009) 747-767, arXiv:0803.2360 [hep-ph].

[29] F. Mahmoudi, "SuperIso: A Program for calculating the isospin asymmetry of B to K* gamma in the MSSM," Comput.Phys.Commun. 178 (2008) 745-754, arXiv:0710.2067 [hep-ph].

[30] S. Heinemeyer, W. Hollik, D. Stockinger, A. Weber, and G. Weiglein, "Precise prediction for M(W) in the MSSM," JHEP 0608 (2006) 052, arXiv:hep-ph/0604147 [hep-ph] .

[31] S. Heinemeyer, W. Hollik, A. Weber, and G. Weiglein, "Z Pole Observables in the MSSM," JHEP 0804 (2008) 039, arXiv:0710.2972 [hep-ph].

[32] M. Verzocchi in talk at ICHEP 2008. 2008, Philadelphia, USA.

[33] ALEPH Collaboration, "Precision electroweak measurements on the $Z$ resonance," Phys. Rept. 427 (2006) 257, arXiv:hep-ex/0509008.

[34] Muon G-2 Collaboration, G. Bennett et al., "Final Report of the Muon E821 Anomalous Magnetic Moment Measurement at BNL," Phys.Rev. D73 (2006) 072003, arXiv:hep-ex/0602035 [hep-ex].

[35] M. Davier, "The Hadronic contribution to (g-2)(mu)," Nucl.Phys.Proc.Suppl. 169 (2007) 288-296, arXiv:hep-ph/0701163 [hep-ph].

[36] Heavy Flavor Averaging Group (HFAG) Collaboration, E. Barberio et al., "Averages of $b$-hadron properties at the end of 2006," arXiv:0704.3575 [hep-ex].

[37] LHCb Collaboration, R. Aaij et al., "First Evidence for the Decay $B_{s}^{0} \rightarrow \mu^{+} \mu^{-}$," Phys.Rev.Lett. 110 (2013) 021801, arXiv: 1211.2674 [hep-ex].

[38] CDF Collaboration, A. Abulencia et al., "Observation of B0(s) - anti-B0(s) Oscillations," Phys.Rev.Lett. 97 (2006) 242003, arXiv: hep-ex/0609040 [hep-ex].

[39] BaBar Collaboration, B. Aubert et al., "Search for the rare leptonic decay $B^{-} \rightarrow \tau^{-} \bar{\nu}_{\tau}$," Phys.Rev.Lett. 95 (2005) 041804, arXiv: hep-ex/0407038 [hep-ex].

[40] P. Chang in talk at ICHEP 2008. 2008, Philadelphia, USA.

[41] HPQCD Collaboration, A. Gray et al., "The B meson decay constant from unquenched lattice QCD," Phys.Rev.Lett. 95 (2005) 212001, arXiv:hep-lat/0507015 [hep-lat].

[42] Particle Data Group Collaboration, C. Amsler et al., "Review of particle physics," Phys. Lett. B667 (2008) 1.

[43] WMAP Collaboration, E. Komatsu et al., "Five-Year Wilkinson Microwave Anisotropy Probe (WMAP) Observations: Cosmological Interpretation," Astrophys.J.Suppl. 180 (2009) 330-376, arXiv:0803.0547 [astro-ph].

[44] ATLAS Collaboration, "Combined measurements of the mass and signal strength of the Higgs-like boson with the ATLAS detector using up to $25 \mathrm{fb}^{-1}$ of proton-proton collision data," ATLAS-CONF-2013-014, ATLAS-COM-CONF-2013-025.

[45] CMS Collaboration, "Combination of standard model Higgs boson searches and measurements of the properties of the new boson with a mass near $125 \mathrm{GeV}, "$ CMS-PAS-HIG-13-005.

[46] F. Feroz and M. Hobson, "Multimodal nested sampling: an efficient and robust alternative to MCMC methods for astronomical data analysis," Mon.Not.Roy.Astron.Soc. 384 (2008) 449, arXiv:0704.3704 [astro-ph].

[47] F. Feroz, M. Hobson, and M. Bridges, "MultiNest: an efficient and robust Bayesian inference tool for cosmology and particle physics," Mon.Not.Roy.Astron.Soc. 398 (2009) 1601-1614, arXiv:0809.3437 [astro-ph].

[48] J. Skilling, "Nested Sampling," in American Institute of Physics Conference Series, R. Fischer, R. Preuss, and U. V. Toussaint, eds., pp. 395-405. Nov., 2004. http://www.inference.phy.cam.ac.uk/bayesys/.

[49] ATLAS Collaboration, "Search for a supersymmetric top-quark partner in final states with two leptons in sqrt(s) $=8$ TeV pp collisions using $13 \mathrm{fb}^{-1}$ of ATLAS data," ATLAS-CONF-2012-167, ATLAS-COM-CONF-2012-202.

[50] ATLAS Collaboration, "Search for direct top squark pair production in final states with one isolated lepton, jets, and missing transverse momentum in sqrts $=8, \mathrm{TeV} p p$ collisions using $21 \mathrm{fb}^{-1}$ of ATLAS data," ATLAS-CONF-2013-037, ATLAS-COM-CONF-2013-038.

[51] ATLAS Collaboration, G. Aad et al., "Search for a supersymmetric partner to the top quark in final states with jets and missing transverse momentum at $\sqrt{s}=7$ TeV with the ATLAS detector," Phys.Rev.Lett. 109 (2012) 211802, arXiv:1208.1447 [hep-ex].

[52] ATLAS Collaboration, G. Aad et al., "Search for direct top squark pair production in final states with one isolated lepton, jets, and missing transverse momentum in $\sqrt{s}=7 \mathrm{TeV}$ pp collisions using $4.7 \mathrm{fb}^{-1}$ of ATLAS data," Phys.Rev.Lett. 109 (2012) 211803, arXiv: 1208.2590 [hep-ex]. 
[53] ATLAS Collaboration, G. Aad et al., "Search for light scalar top quark pair production in final states with two leptons with the ATLAS detector in $\sqrt{s}=7 \mathrm{TeV}$ proton-proton collisions," Eur.Phys.J. C72 (2012) 2237, arXiv:1208.4305 [hep-ex].

[54] ATLAS Collaboration, G. Aad et al., "Search for a heavy top-quark partner in final states with two leptons with the ATLAS detector at the LHC," JHEP 1211 (2012) 094, arXiv: 1209.4186 [hep-ex].

[55] ATLAS Collaboration, G. Aad et al., "Search for light top squark pair production in final states with leptons and $b^{-}$jets with the ATLAS detector in $\sqrt{s}=7 \mathrm{TeV}$ proton-proton collisions," Phys.Lett. B720 (2013) 13-31, arXiv:1209.2102 [hep-ex] .

[56] ATLAS Collaboration, "Search for direct production of the top squark in the all-hadronic ttbar + etmiss final state in $21 \mathrm{fb}-1$ of p-pcollisions at sqrt(s)=8 TeV with the ATLAS detector," ATLAS-CONF-2013-024, ATLAS-COM-CONF-2013-011.

[57] ATLAS Collaboration, "Search for direct top squark pair production in final states with two leptons in $\sqrt{s}=8$ TeV pp collisions using $20 \mathrm{fb}^{-1}$ of ATLAS data.," ATLAS-CONF-2013-048, ATLAS-COM-CONF-2013-056.

[58] ATLAS Collaboration, "Searches for direct scalar top pair production in final states with two leptons using the stransverse mass variable and a multivariate analysis technique in $\sqrt{s}=8 \mathrm{TeV}$ pp collisions using $20.3 \mathrm{fb}^{-1}$ of ATLAS data," ATLAS-CONF-2013-065, ATLAS-COM-CONF-2013-065.

[59] ATLAS Collaboration, "Search for pair-produced top squarks decaying into a charm quark and the lightest neutralinos with $20.3 \mathrm{fb}$-1 of $p p$ collisions at $\sqrt{s}=8 \mathrm{TeV}$ with the ATLAS detector at the LHC," ATLAS-CONF-2013-068, ATLAS-COM-CONF-2013-076.

[60] CMS Collaboration, S. Chatrchyan et al., "Search for supersymmetry in hadronic final states with missing transverse energy using the variables AlphaT and b-quark multiplicity in pp collisions at $8 \mathrm{TeV}$," Eur.Phys.J. C73 (2013) 2568, arXiv:1303.2985 [hep-ex].

[61] CMS Collaboration, CMS, "Search for supersymmetry using razor variables in events with b-jets in pp collisions at 8 TeV," CMS-PAS-SUS-13-004.

[62] CMS Collaboration, CMS, "Search for top-squark pair production in the single lepton final state in pp collisions at 8 TeV," CMS-PAS-SUS-13-011.

[63] ATLAS Collaboration, G. Aad et al., "Search for charginos nearly mass-degenerate with the lightest neutralino based on a disappearing-track signature in pp collisions at $\sqrt{s}=8 \mathrm{TeV}$ with the ATLAS detector," Phys.Rev. D88 (2013) 112006, arXiv:1310.3675 [hep-ex].

[64] ALEPH Collaboration, A. Heister et al., "Search for charginos nearly mass degenerate with the lightest neutralino in e+ e- collisions at center-of-mass energies up to 209-GeV," Phys.Lett. B533 (2002) 223-236, arXiv:hep-ex/0203020 [hep-ex] .

[65] L3 Collaboration, M. Acciarri et al., "Search for charginos with a small mass difference with the lightest supersymmetric particle at $\sqrt{S}$ =189-GeV," Phys.Lett. B482 (2000) 31-42, arXiv:hep-ex/0002043 [hep-ex].

[66] OPAL Collaboration, G. Abbiendi et al., "Search for nearly mass degenerate charginos and neutralinos at LEP," Eur.Phys.J. C29 (2003) 479-489, arXiv:hep-ex/0210043 [hep-ex].

[67] M. El Kheishen, A. Aboshousha, and A. Shafik, "Analytic formulas for the neutralino masses and the neutralino mixing matrix," Phys.Rev. D45 (1992) 4345-4348.

[68] A. Bottino, F. Donato, N. Fornengo, and S. Scopel, "Probing the supersymmetric parameter space by WIMP direct detection," Phys.Rev. D63 (2001) 125003, arXiv: hep-ph/0010203 [hep-ph].

[69] CDMS Collaboration, D. Akerib et al., "Limits on spin-dependent wimp-nucleon interactions from the cryogenic dark matter search," Phys.Rev. D73 (2006) 011102, arXiv:astro-ph/0509269 [astro-ph].

[70] XENON100 Collaboration, E. Aprile et al., "Dark Matter Results from 225 Live Days of XENON100 Data," Phys.Rev.Lett. 109 (2012) 181301, arXiv:1207.5988 [astro-ph.C0].

[71] LUX Collaboration, D. Akerib et al., "First results from the LUX dark matter experiment at the Sanford Underground Research Facility," arXiv:1310.8214 [astro-ph.CO].

[72] XENON1T collaboration Collaboration, E. Aprile, “The XENON1T Dark Matter Search Experiment," arXiv:1206.6288 [astro-ph.IM] .

[73] R. L. Arnowitt and P. Nath, "Loop corrections to radiative breaking of electroweak symmetry in supersymmetry," Phys.Rev. D46 (1992) 3981-3986.

[74] A. Gladyshev, D. Kazakov, W. de Boer, G. Burkart, and R. Ehret, "MSSM predictions of the neutral Higgs boson masses and LEP-2 production cross-sections," Nucl.Phys. B498 (1997) 3-27, arXiv:hep-ph/9603346 [hep-ph]. 